\title{
Die Römische Leiche vom Jahre 1485.
}

\author{
Ein Beitrag zur Geschichte der Renaissance \\ von
}

Henry Thode.

Mit zwei phototypischen Abbildungen.

Onter allen den hervorragenden, für die Geschichte der Kunst so wichtigen Schätzen, welche das Museum Wicar in Lille aufbewahrt, gibt es keinen, der einen gleich tief ergreifenden und nachhaltigen Eindruck hervorbrächte, als die Wachsbüste eines jungen Vädchens, die unter der einfachen Bezeichnung: tête de cire du temps de Raphael im Katalog angegeben ist. Immer von neuem wendet sich der Beschauer von Raphaels Zeichnungen und den von Bildern niederländischer Meister strotzenden Sälen der Galerie zu diesem Wundergebilde zurück, um immer von neuem den räthselhaften Zauber zu empfinden, dem niemand mit Worten Ausdruck zu verleihen und doch niemand sich zu entziehen vermag. Es gibt Werke der bildenden Kunst, die gleichsam musikalisch auf uns wirken und Saiten in unserm Innern berühren, deren Schwingungen jeder Berechnung spotten. Ein solches ist der Liller Kopf und wie ein träumerisches Lied von kaum sich erschliessendem Leben, leise vorgeahntem Liebesglück und frühem, Leben und Liebe knickendem Tod klingt es zu uns herüber, wenn wir in Anschauung versunken ror ihm stehen. Ihu beschreiben hiesse ihn besingen! Wie in einem kurzen Augenblicke zusammengedrängt vereinigen sich zwei Lebensalter auf diesem lieblicben Mädchenantlitz: eben noch Kind, ist kaum ihr selbst bewusst des Lebens Frühling über sie gekommen, und wie sie von heimlichem Ahnen erfüllt demselben nachsinnt, verliert sich träumend der Blick und zieht ein stilles Lächeln um die Lippen. Ein Hauch vou Keuschheit und Unberührbarkeit schwebt um die zarten Züge, 
dem Duft der Blume gleicheud, die sich erschliessen will. Uud doch: über alle diese Lieblichkeit hat sich ein feiner Zug des Leidens gelegt. ein Schatten, wie ihn vicht schnell vorüberziehender Kummer oder Schmerz, sondern nur das Vorgefühl frïhen Todes oder der Tod selbsi hervorruft. Lud spräche nicht der Stoff, aus dem der Kop: geformt. schon genugsam datür, wir wüssten es auch so, dass wir in ihm das Abbild einer Todten seheu, dereu Andenken der Meusch laukbarer als das unerbittliche Geschick bewahrte.

Wer war dies Yädchen, wo lebte, wo starb es uud welches Küustlers Meisterhand war es vergönnt, ihm linsterblichkeit aut Erilen und noch in späten Zeiten die Liebe der Menschen zu erringen? Dis sind Fragen, die noch keine definitive Antwort erhalten haben. Räthselhaft wie der Kopf selbst ist seine Herkunft. Nit zablreichen auderen Werken erbte ihn Lille ron seinem grossherzigen Bürger Jean Baptiste Wicar, der ihn in die Zeit Raphaels setzte, onut anzugeben, wo und ron wem er ihn erworben. Welche Ehre er ihm erzeigte, beweist der Name Raphael, von dessen Autorschatt freilich die meisten späteren Forsther absehen zu müssen glaubteu. Es eutstauden unter ihnen zwei Parteien, von denen die eine einen antikeu Ursprung, die andere eine Entstehung im XV. Jahrhundert fïr die Büste annahm. Wurde die Ansicht der ersteren am bestimntesten von Benvignat ausgesprocken und im Catalog des Musée Wicar autrechterhalten '), so fand (lie zweite, zuerst von Jules Renouvier atufgestellt, einen beredten Interpreten in Louis Gonse. in dessen feinfühliger Analyse und Würdigung des Werkes jeder, der nach ihu sich mit demselbeu beschäftigt, deu wichtigsten Auhaltspunkt für. eigene Forschungen gewinnen wird ${ }^{2}$ ).

Bei einer Beschreibung der Büste ist das l'iedestal und lie (iéwanddraperie als eine Zuthat des letatem Jahrhunderts ausser Acht zu lassen ${ }^{3}$ ).

Der ein wenig unter Naturgrösse gehaltene Kopf von lïuglichem

1) Musée Wicar, Catalogue, Lille 1\$56. S. \$15. 2) Gaz. d. beaux-arts li is. Il P'ir. t. XVIl, S. 19 T ff. Ebds. eine ron Gaillard wit bekannter Mejsterochatt gestochene Reproduction des Kopfes. Photographien desselben bei Braum $\mathrm{Ni}$ 1:s-202. $\left.{ }^{3}\right)$ In geschicktester Weise restaurirt wurde sie, wie (ionse herichtet, durch Herrn Talrich, der bei dieser Gelegenheit fand, dass sie hohl ist und das Wachs eine Stärke von nur einem Centineter besitzt. Sein als eines Sachkenners sehr massgebendes Urtbeil über die Anfertigung geht dahin, dilss das Wachs zuerst in ein hohles Modell von Thon, Gyps oder einer andern Diasse gediürkt, damn mit dem heissen Eisen übergangen und schliesslich gemalt worden ist. 
Oval ist etwas nach links gewandt und gesenkt und der verschleierte Blick der halb geöffneter Augen nach derselben Seite nach unten gerichtet. Die leicbt gewölbte. mittelhohe Stirn ist ron weichem, welligem. in der Mitte gescheiteltem Haare. das sich in dicker Fülle ijber die obere Fläche der Ohreu legt, unrahmt. Die wulstig schwere (Oberaugeupartie trägt wenig geschwungene feine Brauen, zwischen denen die Nase breit ansetzt. Die letztere verläuft ziemlich spitz und zeigt eine geringe, aber charakteristische concave Biegung, die wie Gonse schon bemerkt hat, nichi geuügend in Gaillards Reproduction z.ur Geltung kommt. Die Lage der weit auseinandersteheuden Augen, leren innerer Winkel etwas höher als der äussere liegt, verleiht dem Lopfe jenen siunend träumerischen Ausilruck. Die Waugen sind ziemlich flach, aber weich behaudelt. die Obreu gross, anliegend und in den oberen Theilen durch das Haar niedergedrückt. Die wenig gë̈ffueten Lippen ron entzückenden Wellenlinien sind fein und von einem kaum bemerkbaren Lächeln umspielt. Das Kinn ist breit und rund, der in weichen Biegungen geschwungene Hals fleischig und voll.

Die Haartracht, die Stiru, die Wangen, sowie, ich möchte sagen, der grosse Gesammteindruck der Züge hat etwas so unzweifelhaft Antikes, dass man im ersteu Augenblicke geneigt wäre, Benvignats Ausicht für die richtige zu balten. Eine nähere Besichtigung aber macht sie hinfällig: die Behandlung der Nase, der Augen und der Ohren weist mit Entschiedenheit auf eine Entstehung in der Renaissancezeit und zwar speciell in Florenz hin. Es ist der das kleinste Detail mit voller Liebe durcharbeitende und selbst Zufälligkeiten beriicksichtigende Realismus, der seit Donatello ein Kennzeichen der Florentinischen Sculptur gervordeu und der ihren Werkeu eine so unwiderstehlich fesselnde, von Schöpfungen antiker Kunst so verschiedene Wigenart gibt. Zugleich aber zeigt der Kopf eine derartig freie und kampflose Meisterschaft, eine ausgesprochone, jedweder Herbheit entbehrende Empfindung für Grazie, Aumuth und Weichheit, wie sie erst die zweite, der Vollendung italienischer Kunst nahestehende Periode Florentiner Praxis zeitigte. Aus diesen Gründen kann ich daher Gonse nur beistimmen, wenu er den Meister der Büste in die Nähe des Andrea del Verrocchio und sie selhst in die 80er Jahre des Quattrocento setzt. In dem andereu aber, das die unzweifelhaften antiken Reminiscenzen erklären soll, scheint er mir nicht das Richtige zu treffen. Wohl ist es unbestritteu, dass in der Behandlung der Details die grossen Bahubrecher auf dem Gebiete der italienischen Plastik: Donatello und Ghiberti viel rou der Antike gelernt und dass wir 
deren Einfluss in zahlreichen ihrer Schöpfungen bemerken, aber der Liller Kopf gehört nicht in eine Reihe mit Werken, wie Donatellos Bronzedavid, h. Markus oder Petrus, die frei concipirt, nur im Formellen den Einfluss des Alterthums verrathen, sondern vergleicht sich Sculpturen, die wie Ghibertis von Gonse selbst citirte Köpfe an dessen II. Baptisteriumthüre directe Nachahmungen bestimmter antiker Originale sind, im ganzen den antiken Charakter bewahren, im einzelueu aber mehr oder weniger der individuellen, modernen Formenauffassung des Künstlers entsprechend Veränderungen erleiden '). Kein Zweifel, dass ,eine junge Florentinerin aus der Zeit der Medici einer jungen Römerin aus der Zeit des Augustus gleichen konnte", aber für eine solche frappante Verwandtschaft haben wir in sonstigen Werken des Quattrocento kein Analogon und jeder unbefangen vor den Wachskopf hintretende Beschauer wird sich dem Eindruck nicht entziehen können, das Bildniss einer Römerin vor sich zu haben. Demvach ergibt sich eine Erklärung der künstlerischen Besonderheit der Büste nur aus der Annahme, dass sie eine Florentiner Renaissancearbeit ist, der ein antikes Vorbild zu Grunde liegt. Antik ist der Typus, modern der geistige Gehalt des Kopfes, die Behandlung der Details theilweise modern, theilweise antikisirend.

Betreffs der Frage, welcher Art das Original gewesen, ob ein Marmor-, Bronze-, Thon- oder Wachskopf, scheint das Material der modernen Nachahmung zu Gunsten des letzten zu sprechen, da eine Uebertragung ron Stein oder Metall in Wachs wenig Wahrscheinlichkeit für sich hat und mir unter den aus dem Alterthume erhaltenen Werken keines bekannt ist, das unserer Büste entspräche. War das Vorbild aber aus Wachs, so könnte es nur eine antike Todtenmaske gewesen sein, und diese Meinung würde durch Benvignats Ansicht Bestärkung finden, der im Liller Kopfe eben eine solche zu erkennen glaubte, indem er daraut hinwies, dass derselbe hohl sei und in gewissen Details auf einen Abguss nach der Natur schliessen lasse. Den Beweis, dass sich antike Todtenmasken durch die Jahrhunderte hindurch, selbst bis auf unsere Zeit erhalten konnten, lieferten ihm die Funde in Cumae.

Wiederholt berichten uns die alten Schriftsteller von der römischen Sitte, die Züge der Verstorbenen in Wachs zu verewigen und diese Bildnisse (imagines maiorum) zur Erinnerung im Atrium des Hauses zu bewahren, nachdem sie bei der Bestattung selbst öffentlich

1) Vergl. ein charakteristisches Beispiel publicirt bei E. Müntz: les Précurseurs de la Renaissance. Paris 1881. S. 81. 
gezeigt worden waren. Von neuem geschah dies jedesmal bei dem Leichenzuge eines später Verstorbenen aus demselben Geschlechte, und diese bildliche Begleitung der Ahnen verlieh dem Akte die feierlichste Weihe and brachte den Familienstolz in pomphaftester Weise zum Ausdruck. Das ,ius imaginis" stand anfangs bloss den hohe Aemter bekleidenden Patricierı zu, wurde aber später auch Plebejern, die curulische Würden erlangt, zu Theil 1). Von einem Gebrauch, die vermittelst Gypsabgüssen getreu nach der Natur gefertigten Porträts solchen Todten, welche nicht verbrannt wurden, ins Grab mitzugeben, erfahren wir durch die Schriftsteller nichts, die Thatsache jedoch, dass dies vorkam, ist durch die Funde in Cumae nachgewiesen worden. Es wurden hier in einem Grabe vier Skelette ohue Schädel entdeckt, statt welcher letzteren sich bei zweien Wachsmasken mit Hals und gläsernen Augen vorfanden 2\%. Freilich sind dies die einzigen Beispiele, die sich bis auf unsere Tage erhalten haben, doch würde dies nicht hindern, anzunehmen, dass in der That den italienischen Meister für seine Büste ein solcher antiker Wachskopf als Vorbild gedient haben konnte.

Diese Ansicht dürfte eine scheinbare Bekräftigung finden in einem anderen Werke des $X \nabla$. Jahrhunderts, das schon von verschiedenen Forschern in Beziehung zum Liller Kopfe gebracht worden ist: der schönen, wie jener dem Raphael zugeschriebenen weissgehöhten Kreidezeichnung in der Albertina in Wien, die den lieblichen Kopf eines etwa dreizehn- bis fünfzehnjährigen Mädchens zeigt. Derselbe, am Halse abgeschnitten, liegt halb nach rechts gewandt in fast aufrechter Stellung auf einem durch eine Quaste deutlich als solches gekennzeichneten Kissen, das seimerseits in einer Art starken Holzteller oder -scheibe (vielleicht einer Modellirscheibe) seine Unterlage findet. Das Auge ist nur wenig geöffnet und die Ruhe des Todes breitet sich über die Züge aus ${ }^{3}$ ). Trotz maucher Verschiedeuheiten im einzelneu ist der Gesammteindruck ein demjenigen der Büste so verwandter,

1) Vergl. Iuvenal, sat. VIII, 2. Ovid, Fast. I. 591. Polybius VI, 53. Tacitus, Ann. IV, 9. III, 76. Plinius, hist nat. XXXV, II. Varro V, XXI. - Vergl. auch Spire Blondel: les modeleurs en cire. Gaz d. b. a. Il. pir. t. XXV, S. $500 \mathrm{ff}$. 2) Vergl. Minervini: bulletino arch. Napolitano N. 14 Januar 1858. Raoul. Rochette: revue archéologique IX, II, S. $770 \mathrm{ff}$. Die Masken befinden sich jetzt im Museum zu Neapel. ${ }^{3}$ ) Die Zeichnung ist, wie wir dies in unserer phototypischen Abbidaung durch eine stärkere schwarze Linie angezeigt haben, oben halbrund abgeschnitten derart, dass nur noch die Stirne, nicht aber das Haar: über derselben mehr sichtbar ist. Ëin späterer Ergänzer hat, um den Schaden wieder gut zu machen, ein schleierartiges Tuch hinzugefügt, das lose äber dem hopfe liegt. 
dass man für beide dasselbe Vorbild annehmen muss, aber auch nur das Vorbild, da die Zeichnung den Liller Kopf selbst nicht reproducirt. Das zeigen die $\mathrm{Abweichungen} \mathrm{auf} \mathrm{das} \mathrm{deutlichste:} \mathrm{das} \mathrm{Gesichts-}$ oval erscheint nicht so lang, die Augen sind von den schweren Lidern fast geschlossen, die Krümmung der Nase ist, wenn auch bemerkbar. doch nicht so stark hervorgeboben uud das Haar liegt nicht in so weichen Wellenlinien in die Stirne hinein und über das Ohr hiu. Man möchte sagen, der antike Charakter äussere sich in der Zeichnung noch mehr als in der Sculptur. So scheint das Blatt die oben ausgesprochene Vermuthung zu bestätigen und wie die Büste eine antike Maske wiederzugeben ${ }^{1}$ ).

Und doch, so viel Wahrscheinlichkeit die Anuahme für sich hat, so ist sie gleichwohl nicht sicher, da verschiedene Anzeichen eher darauf hinweisen, dass die Zeichnung nicht nach einem plastischen Originale, sondern vach der Natur gemacht wurde. Zunächst kann die starke Zusammenziehung des offenbar ziemlich compacteu Kissens nur durch eine schwere Last, wie sie eine Wachsmaske nicht ist, bewirkt werden und deutet an, dass der Gegenstand, der sie hervorbrachte rund und körperlich war, wie der wirkliche Hals eines Menschen. Ferner ist das Haar in so natürlich lockerer, ja etwas verworrener Weise und derart im einzelnen wiedergegeben, wie es an irgend einem plastischen Werke, sei es von welchem Materiale es wolle, nie zu sehen ist. Man vergleiche nur mit der Zeichnung selbst die weiche, aber bloss im grossen andeutende Haarbehandlung der Büste, um den Unterschied zwischen Natur und Wachsimitation deutlich zu gewahren. Immerhin kann es zweifelhaft bleiben, ob das Vorbild nicht doch eine Maske war und der Künstler das Haar flüchtig skizzirend dasselbe natürlicher gestaltete, als er es vor Augen hatte. Zeichnete er aber in der That nach einem vou seinem Körper getrennten Kopfe, so widerspricht dies auf der anderen Seite dem sus der Betrachtung der Büste gewonnenen Resultate, dass dieselbe auf ein antikes Vorbild zurückgeht! Also wäre Louis Gonse doch im Recht und die Verwandtschaft mit der Antike nur eine zufällige? Oder dürften wir an eine andere Lösung des Räthsels glauben?

1) Für die Annahme, dass beide Werke dasselbe Vorbild hatten, spricht vielleicht auch noch der Umstand, dass am Halse der Büste genan an derselben Stelle, an welcher der Hals auf der Zeichnung abgeschnitten ist, eine feine rissartige Linie bemerkbar ist, die vermuthen lässt, dass der Bildhauer zuerst den Kopf nur bis so weit ausgeführt und erst dann ergänzend zur Büste gestaltet habe. 
In seiner "Cultur der Renaissance" erzählt Jacob Burckhardt von einem wunderbaren Ereignisse, das im Jahre 1485 ganz Rom in die grösste Aufregung rersetzte 1). ,Bei dieser Stimmung der Gemüther geschah es am 15. April 1485, dass sich das Gerücht verbreitete, man habe die wunderbar schöne, wohl erhaltene Leiche einer jungen Römerin aus dem Alterthume gefundeu. Lombardische Maurer, welche auf einem Grundstück des Klosters S. Maria Nuova, an der Via Appia ausserhalb der Caecilia Metella, ein antikes Grabmal aufgruben, fanden einen marmornen Sarkophag angeblich mit der Aufschrift: Julia, Tochter des Claudius. Das Weitere gehört der Phantasie an. Die Lombarden seien sofort verschwunden sammt den Schätzen und Edelsteinen, welche im Sarkophag zum Schmuck und Geleit der Leiche dienten; letztere sei mit einer sichernden Essenz überzogen und so frisch, ja so beweglich gewesen wie die eines eben gestorbenen Mädchens ron 15 Jahren; dann hiess es sogar, sie habe noch ganz die Farbe des Lebens, Augen und Mund halb offeu. Man brachte sie nach dem Conservatorenpalast auf dem Capitol, und dahin, um sie zu sehen, begann nun eine wahre Wallfahrt. Viele kamen auch um sie abzumalen; , denn sie war schön, wie man es nicht sagen noch schreiben kann, und wenn man es sagte oder schriebe, so würden es, die sie nicht sahen, doch nicht glauben." Aber auf Befehl Innocenz VIII. musste sie eines Nachts vor Porta Pinciana an einem geheimen Ort verscharrt werden; in der Hofhalle der Conservatoren blieb nur der leere Sarkophag. Wahrscheinlich war über den Kopf der Leiche eine farbige Maske des idealen Stiles aus Wachs oder etwas Aehnlichem modellirt, wozu die vergoldeten Haare, von welchen die Rede ist, ganz wohl passen würden. Das Rührende an der Sache ist nicht der Thatbestand, sondern das feste Vorurtheil, dass der antike Leib, den man endlich hier in Wirklichkeit vor sich zu sehen glaubte, nothwendig herrlicher sein müsse, als alles was jetzt lebe."

Sollten wir in diesem Vorfalle Aufschluss über die Entstehung der Liller Büste und der Wiener Zeichnung finden und sollte die jugendliche Römerin, deren Bildniss wir in beiden bewundern, eben jenes Mädchen sein, das in so wunderbarer Weise nach langer Todesruhe und Vergessenheit wieder ans Licht gestiegen allen humanistischen und künstlerischen Kreisen jener Zeit als Symbol der Auferstehung des Alterthumes selbst erscheinen mochte? Wäre dem wirklich so, dann hätten wir eine schlagende Erklärung für den aus an-

1) III. Auflage von L. Geiger. Bd. I, S. 230.

Mitthellungen IV. 
tiker und moderner Formenanschauung so eigenartig gemischten Stil, eine Erklärung dafür, dass die Büste bald dem Alterthume, bald der Renaissancekunst zugeschrieben werden konnte, eine Erklärung endlich für den räthselhaften Eindruck, den sie auf jedermann hervorbringt. Die Frage scheint eine abermalige, eingehende Beschäftigung mit deu literarischen Quellen, aus denen Burckhardt gescböpft, werth. Es sind deren drei.

I. verzeichnet Nantiporto in seinem Diarium Romanum ") das Ereigniss unter dem Jabre 1485 folgendermassen: „Am 18. (i. e. April) wurde auf einem Grundstück (des Klosters) S Maria Nuova oberhalb des Capo di Bove ein vollständiger Leichnam in einem Marmorsarkophage gefunden. Am Dienstag d. 19. wurde besagter Leichnam in den Conservatorenpalast getragen und es gingen so viele Leute hin, ihn zu sehen, dass es den Anschein hatte, als ob Ablass dort ertheilt würde. Und er wurde in einen Kasten von Holz gelegt und blieb unbedeckt stehen. Es war ein jugendlicher Leichnam, dem Aussehen nach etwa 15 Jahre alt und es fehlte ihm kein Glied. Seine Haare waren schwarz, als wäre er kurz zuror gestorben. Er hitte eine Mixtur, welche ihn, wie man sagte, mit den weissen Zähuen, der Zunge und den Augenbrauen conservirt hatte. Man weiss nicht gewiss, ob er männlichen oder weiblichen Geschlechtes gewesen. Viele glauben, er sei im Jahre 170 gestorben."

II Mit mehr Ausführlichkeit behandelt Stephanus Infessura in seinem Diarium den Vorfall ${ }^{2}$ ): „An demselben Tage (welcher, ist im vorhergehenden: mense martii sequentis anni $148 j$ nicht genau be-

1) Diarium Roma num urbis ab anno 1481 ad 1492 autore anolıymo synchrono Notario de Nantiporto bei Muratori, rer. Ital. script. III, pars II, col. 1094 : Alli 18. fu trovato in un casale di S. Maria Nuova sopra Capo di Bove un corpo intero in un pilo di marmo Alli 19. martedi fu portato il detto corpo in casa de' Conservatori e andava tanta gente a vederlo, che pareva vi fosse la perdonanza. E fu messo in una cassa di legname e stara scoperto. Era corpo giovenile, mostrava da quindici anni, non gli mancava membro alcuno, aveva i capelli negri, come se fusse morto poco prima. Aveva una mistura, la quale si diceva lareva conservato, co i denti bianchi, la lingua, le ciglia Non si sa certo, se fusse maschio o femmina. Molti credono, sia stato morto degli anni 170 .

2) Stephani Infessurae senatus populique Romani scribae diarium urbis Romae. Mangelhaft publicirt bei J. G. Eccard, Corpus hist. medii aevi II, col. 1951, dann besser durch Muratori im II. Theile des III. Bandes seines citirten Werkes, dem ich hier folge: Eadem die Fratres et Conventus Sanctae Mariae novae fodi faciebant in quodam eorum casali posito extra portam Appiam in via Appia distanti ab urbe per quinque vel sex milliaria. Et cum prope viam rel in ipsa via a fundamentis quoddam sepulcrum destruxissent, in ultimo loco 
stimmt) liessen die Brüder des Klosters S. Maria nuova auf einem ihnen zugehörigen Grundstück, das ausserhalb der Porta Appia an der Via Appia etwa fünf oder sechs Viglien von der Stadt entfernt liegt, graben. Und als sie nahe bei der Strasse oder auf der Strasse selbst ein gewisses Grabmal bis auf die Fundamente zerstört hatten, fanden sie an der tiefsten Stelle des Fundaments eine Marmortruhe, die mit einem Marmorsteine röllig bedeckt und mit Bleiverschluss verseben war. Als sie denselben geöffnet hatten, fanden sie den unversehrten Leichnam einer Frau, der mit einer Geruch verbreitenden Mixtur überzogen war, eine Art goldner Haube oder Inful auf dem Haupte, goldene Haare rings um die Stirne und fleischige röthliche Wangen hatte, als ob sie noch jetzt Leben habe. Die Augen und ähnlich der Mund waren ein wenig geöffnet und die Zunge liess sich fassen und aus dem Munde herausziehen und kehrte dann unaufhaltsam

fundamenti quamdam cassam marmoream coopertam marmoreo lapide implumbatam invenerunt. Quam cum aperuissent, unum corpus cuiusdam mulieris integrum invenerunt involutum quadam odorifera mixtura cum quadam cufia seu infula aurea in capite et cum capillis aureis circum circa frontem et cum carne et rubore in maxillis, acsi etiamnum viveret. Habebat oculos parum apertos et os similiter, et lingua capiebatur et extrahebatur ex ore et redibat in continenti ad locum suum. Deinde ungulae manuum et pedum firmissimae ac albae et bracchia levabantur et redibant ad locum suum, ac si nunc mortua fuisset, stetitque per multos dies in Palatio Conservatorum, ubi propter aërem colorem tantum faciei mutarit factaque fuit nigra, sed non propterea pinguedo vel caro destructa erat. Cumque Conservatores in eodem pilo ad locum iuxta Cisternam in reclaustro eiusdem Palatii posuissent, a dicto Innocentio iussi in locum incognitum de nocte extra Portan Pincianam in quodam vico vicino ejus, ubi fovea defossa fuerat reportaverunt ibique eam sepeliverunt. Et creditur fuisse corpus Juliae Ciceronis filiae. Et illis primis diebus quibus inventa est, ad dictum palatium inducta fuit, tantus erat concursus hominum eam videre cupientium, ut passim in platea. Capitolii vendentes olia et alia ad instar fori reperirentur. Et mixturam odoriferam cum qua involuta erat, ferebatur confectam esse ex myrrha et olibano, alii ferunt Aloë Tribentina (bei Eccard richtiger: Therebintina, von beiden missverstanden als Adjectivum betrachtet), quae acutissimum et quodam modo obstupefactivum habebat odorem. Et multi existimaverunt cum ea repertam fuisse maximam auri quantitatem vel argenti et lapidum pretiosorum. Quod existimatum fuit ex eo, quod fodientes et qui super eos erant numquam fuerunt ulterius reperti. Et, aetatis suae erat, ut videri poterat, duodecim rel tredecim annorum. Et erat adeo pulchra et formosa, quod vix scribi seu dici posset, et si diceretur aut scriberetur, a legentibus qui eam non viderunt minime crederetur. Et multi de longinquis partibus venerunt causa videndi eam et depingendi eius pulchritudinem et non potuerunt eam videre, quia fuerat, ita ut supra scriptum est, secreto in loco proiecta. Et ita male contenti recesserunt. Et pilus marmoreus, ubi fuerat reperta, remissus ost in reclanstro dominorum Conservatorum. 
an den früheren Ort zurück. Dann waren auch die Nägel der Hände und Füsse sehr hart und weiss und die Arme hob man in die Höbe und sie kehrten in ihre Lage zurück, als ob sie eben jetzt gestorben wäre. Cnd sie blieb viele Tage hindurch im Conservatorenpalast stehen, wo sie in Folge der Luft die Gesic htsfarbe so veränderte. dass sie schwarz wurde. Gleichwohl wurde desswegen nicht die Fettschicht noch das Fleisch zerstört. Und als die Conservatoren sie in demselben Sarkophag an einen Ort nahe bei der Cisterne in dem Kreuzgange desselben $\mathrm{Pa}$ lastes hingestellt hatten, mussten sie auf Befehl des genannten Innocenz sie bei Nacht an eine unbekannte Stelle ausserhalb der Porta Pinciana in einem nahe bei derselben gelegenen Flecken, wo eine Grube gegraben worden war, tragen und daselbst begraben. Ond man glaubt, es sei der Leichnam der Julia, Tochter des Cicero, gewesen. Und während jener ersten Tage, in denen sie gefunden und nach besagtem Palaste übergeführt worden war, fand ein so grosser Zusammenlauf ron Menschen statt, die begierig waren sie zu sehen, dass weit und breit auf dem Capitolsplatze wie auf einem Markte Verkäufer von $\mathrm{Oel}$ und anderen Dingen zu finden waren. Und es ging die Rede, dass die stark riechende Mixtur, mit der sie umzogen war, aus Myrrhen und Olivenöl verfertigt gewesen sei, andere behaupten aus Aloe und Terpentinöl, das einen sehr scharfen und in gewisser Weise betäubenden Geruch besass. Ond viele waren der Meinung, mit ihr sei eine sehr grosse Menge Goldes, Silbers und kostbarer Steine gefunden worden, was man daraus schloss, dass die. welche ausgegraben hatten, und ihre Aufseher niemals wieder zu finden waren. Und ihr Alter, wie man hatte sehen können, war 12 oder 13 Jahre. Und sie war so schön und wohlgestaltet, wie man es kaum beschreiben oder sagen kann, und wenn man es sagte oder schriebe, würden es doch die Leser, die sie nicht gesehen, nimmer glauben. Und viele kamen von entfernten Gegenden, um sie zu sehen und ihre Schönheit abzumalen und konnten sie nicht sehen, weil sie, wie oben beschrieben wurde, an einen geheimen 0rt weggeworfen worden war, und mussten so schlecht befriedigt heimkehren. Und der Marmorsarkophag, in dem man sie gefunden hatte, wurde in die Hofhalle der Herren Conservatoren zurückgestellt."

Die Quelle III befindet sich in der Chronik Perugias von F. Matarazzo, der in seiner Schilderung von Karls VIII. italienischem Heereszuge in der Absicht, "seinen Geist zur Erholung sich etwas ergehen zu lassen und seinen Lesern eine angenehme Abwechslung zu bieten, sich unterbrechend die Begebenheit folgendermassen be- 
richtet 1): (Indem ich Euch erzähle) wie im Jahre 14.. (leere Stelle im Manuscript) in der alten Stadt Rom ein Grabmal von lombardischen Maurern gefunden wurde, in welchem sich eine wohlgestaltete und anmuthige Jungfrau von Namen Julia, wie das Epitaph zu lesen gab, befand. Ond ihr blondes Haupthaar war mit einem aus vielen und sehr reichen kostbaren Steinen bestehenden Diadem geschmückt und ihre goldenen Haare waren mit einer Binde von grüner Seide gebunden Besagtes Diadem und andere zahllose Kleinodien wurden von jenen Maurern weggenommen, die sobald sie das Mädchen gefunden, „Fersengeld gaben ". Und dieser Leichnam war in eine sehr grosse Fülle von Flüssigkeit gelegt, welche Flüssigkeit sein Fleisch fleckenlos erhalten hatte, so dass es den Anschein hatte, als sei sie kaum gestorben. Cnd das Denkmal war ron grosser Schönheit und ein Theil jenes Epitaphs lautete so: Julia filia Claudi. *

Aus einer Vergleichung der drei Berichte ergibt sich zunächst, dass keiner von dem andern abhängig ist und dass, was Genauigkeit und Ausführlichkeit der Schilderung anbetrifft, Infessura den Vorrang hat. Bei Matarazzo ist die Geschichte zur Anekdote geworden und demgemäss ausgeschmückt und mit der deutlichen Absicht Eindruck zu machen, mit scherzhaftem Anstriche vorgetragen, was aus der Wichtigkeit, mit der das Nebensächliche, der Diebstahl der Kostbarkeiten, behandelt wird, hervorgeht. Der Mangel der Jahreszahl und das Absehen von einer detaillirten Beschreibung der Leiche sprechen dafür, dass er das Ereigniss nur von Hörensagen kennt, möglich jedoch, dass er den im Conservatorenpalast verbliebenen Sarkophag selbst gesehen hat. Seine Angaben sind also mit Vorsicht aufzunehmen. Von grösserem Interesse ist der Bericht Nantiportos, welcher den Vorfall ohne gerade eine wärmere und tiefere Theilnahme für ihn zu haben, in ziemlich trockner und erledigender Weise registrirt, über den Fundort und das Datum aber wohl unterrichtet

1) Cronaca della città di Perugia dal 1492 al 1503 di Francesco Matarazzo detto Maturanzio, publ. im Archivio storico Italiano I. Serie t. XVI, II, p. 180: Commo nell' anno 14 . ( (acuna nel M. S.) fo trovato nell' antica città de Roma una sepoltura da certi lombarde e moratore (sic!) nella quale era una formosa e pulita Giovane chiamata Julia, commo per epitalfio se leggieva; e era adornata sua trezza bionda de molte e ricchissime pietre preziose in una corona in testa, e erano suoi chiome d'oro ligate cum una bendella de seta verde: la quale corona e altre gioje infinite furno tolti per quelli muratore, subbito che l'ebbono trovata fecerunt a te levavi. Et era questo corpo morto in una grandissima copia de liquore, dal quale liquore era conservata sua carne immaculata che appena pareva morta; e era el monumento de gran bellezza e parte de quello epitaffio diceva cosi: Julia filia Claudi. 
ist und als Augenzeuge wohl selbst anf dem Capitol gewesen sein mag, aber freilich als ein von dem Enthusiasmus der anderen kalt gelassener Beobachter. Gerade deswegen sind seine Angaben von Wichtigkeit. Augenzeuge war wohl auch Infessura, wie dies die Begeisterung, mit der er schildert, und die Ausführlichkeit, mit der er zahlreiche von Nantiporto unerwïhnt gelassene Details angiebt, zu beweisen scheinen. Da er den Diebstahl vieler mit dem Leichnam gefundener Kleinodien nur als Gerücht erwähnt und sich auch sonst, wie das wiederholte: "man glaubt, man sagt " zeigt, in seinem Trtheil zurückhält, ist man wobl berechtigt ihm in dem, was er bestimmt versichert, Glauben zu schenken. Dann aber hat sich offenbar Burckhardt den Berichten gegenüber zu skeptisch verhalten und vieles auf das Gebiet der Phantasie verwiesen, was in den Bereich der Thatsachen gehört. Versuchen wir die letzteren auszuscheiden.

Bezüglich des Datums der Auffindung der Leiche zeigen sich bei I und II Verschiedenheiten, doch verdient Nantiportos genaue Angabe mehr Glauben und darf als die richtige betrachtet werden.

Im Fundorte stimmen sie beide überein, nur dass Infessura ihn näher präcisirt, wohingegen uur Matarazzo davon weiss, dass es lombardische Maurer gewesen, welche den Leichnam gefunden, eine Behauptung, die auf einer glaubwürdigen Tradition beruben mag. Den von Nantiporto und Matarazzo erwähnten Sarkophag beschreibt Infessura genauer, ebenso die Mixtur, die bei Matarazzo unbestimmter eine Flüssigkeit genannt wird. Bei allen ist es ein unversehrt wie lebendig conservirter Körper, für dessen erstaunliche Erhaltung Infessura in der Beweglichkeit der Glieder eigenthümliche, sicher nicht auf Erfindung beruhende Beweisgründe anführt. Dem Anscheine nach 12-1j Jahre alt, und zweifellos weiblichen Geschlechtes, da nur der wenig interessirte Nantiporto, der auch das Epitaph nicht kennt, darüber im Unklaren ist. Im Widerspruch zu den beiden andern, die von goldenem oder blondem Haare sprechen, nennt der letztere dieselben schwarz, erwähnt seinerseits aber besonders die gute Erhaltung der weissen Zähne und der Augenbrauen. Aus der Nebeneinanderstellung ron trezza bionda und chiome d'oro bei Matarazzo geht hervor, dass oro hier, wie aureus bei Infessura bildlich als goldig blond zu verstehen ist. Die goldene Haube oder Infula des Infessura hingegen ist bei Matarazzo eine Binde von grüner Seide. Die bestimmte Angabe einer Veränderung der Fleischfarbe durch die Luft bringt nur Infessura. Nur Nantiporto als trockner Chronist stimmt nicht in die begeisterte Schilderung der Schönheit des Mädchens mit ein, weiss aber auch 
von dem ungewöhnlichen Zusammenlauf der Menschen auf dem Capitol zu erzählen.

Von den weiteren Schicksalen der Leiche berichtet bloss Infessura und vermittelt die allgemeine Annahme, dass in ibr Ciceros Tochter zu sehen sei. Dass diese Taufe wenigstens nicht ganz auf Erfindung beruht, beweist Matarazzo, der ron einem Epitaph, in welchem Julia filia Claudi zu lesen war, spricht und hier zweifellos gensuer unterrichtet ist als die anderen. Kann doch nur aus dem Vorhandensein des Namens Julia auf dem Sarkophag jene erste Namengebung erklärt werden; möglich, dass auch das von Nantiporto erwähnte Jahr 170 mit in der Inschrift, von welcher nach Matarazzo die Worte Julia filia Claudi ja nur einen Theil bildeten, enthalten war.

Demnach lässt sich aus den verschiedenen Erzählungen in Kürze gefasst Folgendes als historischer Kern herausschälen: am 18. April 1485 wurde auf einem Grundstück des Klosters S. Maria nuova an der Via Appia jenseits des Grabmals der Caecilia Metella von lombardischen Maurern in einem Grabmal ein hermetisch verschlossener Marmorsarkophag gefunden, in dem sich eine durch eine eigenartige Mixtur in wunderbar lebendiger Weise erhaltene Leiche eines jungen 12 bis 15jährigen Mädchens befand, deren Name als Julia filia Claudi in einer Inschrift angegeben war. Sie hatte blondes, durch eine Art Haube oder Binde gehaltenes Haar, etwas geöffnete Augen und Mund, weisse Zähne, Lebensfarbe und Fleisch in den Wangen und noch bewegliche Glieder. Am anderen Tage auf dem Capitol öffentlich ausgestellt, erregte sie das grösste Aufsehen nicht allein in Rom, sondern auch in entfernteren Gegenden und veranlasste eine förmliche Wallfahrt, bis sie unter dem Einflusse der Luft schwarz geworden, auf Befehl Innocenz' VIII, dem wohl der mit ilir getriebene, fast heidnische Cultus ärgerlich geworden war, ausserhalb der Porta Pinciana heimlich verscharrt wurde ${ }^{1}$ ).

Die wunderbare Erhaltung der Leiche, so räthselhaft sie er-

1) Nibby (diutorni di Roma II, S. $₹ 74$ f.) glaubte in einem Sarkophage des Capitolinischen Museums mit der Aufschrift: M. M. Aurel Extricate, denjenigen der Julia wiederzuerkennen, mit Unrecht, wie schon das Epitaph lehrt und wie es im nächst erscheinenden 2. Theil des VI. Bandes des Corpus Inscriptionum Latinarum unter N. 18310 nachgewiesen wird. Ob derselbe sich überhaupt erhalten, ist bis jetzt noch nicht ermittelt und es scheint auch unter dem für das eben citirte Werk gesammelten Material keine auf Julia filia Clandi lautende Grabinschrift vorzukommen. Ich verdanke diese Mittheilungen der Güte des Herrn Dr. Hülsen in Rom. 
scheinen mag, spielt doch eine so grosse Rolle bei dem ganzen Ereigniss und wird bis ins Einzelne hinein so genau geschildert, dass sie keine Ausgeburt der Phantasie jener in ihren Diarien gewissenhaft die Zeitvorkommnisse verzeichnenden Schriftsteller, sondern nur historisches Factum sein kann. Mehr dazu berufenen Kräften muss es überlassen bleiben, eine naturwissenschaftliche Erklärung dafür zu finden - durchaus unglaublich ist der Vorfall nicht. Erzählen doch Georges Perrot und Charles Chipiez in ihrer ,Geschichte der Kunst des Alterthums "1) ron dem Funde der Mumie einer hochgestellten Dame aus der Ramessidenzeit, die „nach Ablösung der leichten Linnenhülle und der ringsherum gewundenen Streifen so vor uns dalag, als sei sie unmittelbar aus der Taricheutenwerkstatt in Memphis gekommen. Sie trug schwarzes, in dünne Zöpfe geflochtenes Haar; zwischen den etwas verzerrteu Lippen war noch jeder Zahn an seiner Stelle, die schmalen Finger- und Zehennägel waren mit Henna gefärbt. Die Glieder liessen sich noch biegen und die Körperformen, deren hier und da scheinbar noch schwellende Haut röllig fest und glatt anlag, waren fast unverändert." Die Fleischfarbe allerdings hatte sich nicht erbalten, sondern das Aussehen von , verkohltem Papier oder getheertem Segeltuch " angenommen ${ }^{2}$ ). So kann uns also die Beweglichkeit der Glieder und Erhaltung der Formen an der römischen Leiche, die nicht wie jene Aegypterin dreitausend Jahre unter der Erde gelegen, nicht allein als möglich, sondern als gewiss erscheinen und es gälte nur, auch für die Ursprünglichkeit der Fleischfarbe ein Analogon oder eine Erklärung zu finden, um jene Berichte für durchaus wahrheitsgetreu zu halten. Vielleicht liesse sich die Thatsache aus einer besonderen Beschaffenheit der Luft oder aus dem hermetischen Verschluss des Sarkophages erklären ${ }^{3}$ ).

Eine eigentliche Mumie scheint, da durchaus keine Erwähnung von einer Umwickelung mit Binden oder Hüllen geschieht, der Leichnam nicht gewesen und die Mirtur, deren Ingredientien: Olivenöl Myrrhen, Aloe und Terpentinöl allerdings an die beim Einbalsamiren von den Aegyptern angewandten erinnern, nur äusserlich verwerthet,

1) Deutsche im Erscheinen begriffene Ausgabe von Dr. R. Pietschmann. Aegypten, 4. Lief. S. 159 f. ${ }^{2}$ ) Vergl. auch den a. a. O. wiederholten Bericht Passilacqua's über die Entdeckung der Mumie eines jungen Weibes in Theben. 5) Ich erinnere hier an den berübmten Bleikeller des Domes in Bremen, in dem sich, wie bekannt, durch Jahrhunderte hindurch Leichname erbielten, obne der Verwesung anheimzufallen. Die Gebeine derselben sind wie mit Pergament überzogen, Zabne, Haare, Naggel sind geblieben. Die Farbe ist weiss und man kann beim Anblick dieser Körper bei weitem nicht an Mumien denken. Vergl. Storck, Ansichten von Bremen. Frankfurt a. M. 1822. S. 250 f. 
worden zu sein. Fällt die Bestattung der Jalia thatsächlich in das Jahr 170 (n. C.), so würde die Art derselben einen weiteren interessanten Beleg dafür liefern, wie weit aegyptische Sitte zu jener Zeit in Rom um sich gegriffen hatte.

Für uns handelt es sich in erster Linie darum, ob durch jene sichernde Mixtur auch der Kopf selbst lebensvoll erhalten war oder an seiner Stelle, wie Burckhardt glaubt, eine Wachsmaske sich befand. Da ist nun zunächst zu betonen, dass Infessura bei der Genauigkeit seiner Schilderung schwerlich die Erwăhnung einer solchen übergangen haben sollte. Was er und die anderen Quellen sagen, widerspricht dem aber geradezu: die Beweglichkeit der Zunge, die durch die Luft bewirkte Veränderung der Gesichtsfarbe, die weissen Zähne. Der Ausdruck: gold ene Haare schliesslich kann, wie schon bemerkt wurde, auch bildlich oder als künstlich vergoldet gefasst werden und ist selbst im eigentlichen Sinne genommen nicht beweisfähig genug, die gegen die Annahme einer Wachsmaske sprechenden Gründe zu entkräften. Wie der Körper war also auch der Kopf in äberraschender Weise conservirt und die begeisterte Schilderung der Schönheit bezieht sich wohl in Sonderheit auf ihn. Eine leise Uebertreibung derselben wird man gern dem Enthusiasmus und der Verwunderung der Augenzeugen zu Gute halten 1).

Ist das ganze Ereigniss aber nun Thatsache, sollte es da zu kïhn

1) Von Seiten eines dem Verfasser nahe befreundeten Botanikers erhalten wir folgendes Gutachten über die Wahrschein lichkeitsfrage der Conservierung: , Wenn man bedenkt, was für conservierende Flüssigkeiten den Alten um 170 p. Ch. n. zu Gebote standen, so ist das Allerwahrscheinlichste, dass der betreffende Leichnam in Olivenöl, dem Oel der Olea europaea L. aufbewahrt worden sei. Welche Substanzen dem Oel beigesetzt wurden, lässt sich selbstrerständlich heute nicht ermitteln. Es ist aber sebr plausibel, dass in dem Oele ein Quantum Terebinthenharz (ron Pistacia Terebinthus L.) gelöst worden ist. Durch diesen Process wurde das Oel zugleich dickflüssig und seine Dickflüssigkeit hatte entschieden wäbrend der jahrhundertelangen Einkapselung in dem Sarkophage noch zugenommen. Für diesen Aggregatzustand würde auch der stets wiederkehrende Ausdruck , mixtura e vollkommen gerechtfertigt sein, Es ist sehr leicht denkbar, dass man die Leiche nicht in flüssigem Oel, sondern in zăhflüssiger geléeartiger Masse fand. Der penetrante Geruch kann auf Terebinthenharzbeimischung, aber auch auf den Beisatz anderer aromatischer Pflanzen oder Pflanzentheile zurückgeführt werden. Die Leiche dürfte dann insofern à la Mumie präpariert gewesen sein, als man die Baucheingeweide herausgenommen und die Eöhlung des Alvus mit Harzen und aromatischen Substanzen gefüllt hatte, denn zujener Zeit kannten die Römer den ganzen Apparat des altägyptischen Leichencultes; sie kannten Weihrauch, Myrrhe und auch Aloë. Kurz: Beruhen die Berichte auf wahren Thatsachen, so ist die Conservierung in Olivenöl, dem allerhand Harze und aromatische Substanzen zugesetzt gewesen sein mögen, die wahrscheinlichste." Die Red. 
sein, die zwei modernen Kunstwerke, deren Entstehung wir aus dem Stile heraus in den Ausgang des XV. Jahrhunderts verlegen mussten, auf dasselbe zu beziehen? Beide zeigen ans das Bildniss eines jungen Mädchens von antik römischem Typus im Alter von etwa 14 oder 15 Jahren und beide sind nach einem antiken Vorbilde und zwar dem Kopfe einer Todten gefertigt worden, beide nur eine etwas verschiedene Interpretation desselben zeigend. Lnd selbst die goldig blonde Haarfarbe der Julia findet sich auf dem Liller Wachskopf wieder, der gleich nach seiner Vollendung, als das Wachs noch weich war, in lichten feinen Tönen mit dem Pinsel gefärbt wurde. Zu weit gehen hiesse es freilich, wollte man aunehmen, dass diese Färbung durchweg getreu dem Originale entlehnt sei, vielmehr mag der Künstler in derselben mehr oder weniger seinem Ideale nachgegangen sein, wie er ja auch offenbar in der plastischen Nachbildung seine Individualität nicht verleugnen konnte. Getreuer dürften die Züge der Todten uus vielleicht in der Zeichnung aufbewahrt sein, mag dieselbe nun nach dem rom Körper getrennten Kopfe selbst oder, was nun allerdings fast glaubwürdiger klingt, nach einem von demselben genommenen Gypsabgusse gefertigt sein.

Viele Künstler kamen, wie Infessura erzählt, vergeblich in der Absicht nach Rom, die wunderbar zum Scheinleben Wiedererweckte abzumalen - von denen aber, die daselbst lebten, werden es wohl wenige gewesen sein, die nicht auf die Kunde des Vorfalls eiligst zum Capitol gewandert wären und Dank ihrer Kunst das bildliche Andenken an die liebliche Todte mit nach Hause genommen hätten. Wer weiss, ob sich nicht dereinst ausser der Liller Büste und der Wiener Zeichnung noch andere Beweise dafür werden finden lassen? Wer die Meister jener beiden Werke gewesen, ist, wie gesagt, mit Sicherheit noch nicht ermittelt worden, doch lohnt es hier darauf zurückzukommen, da wir jetzt einen festeren Anbaltspunkt für das Erfassen der Individualität des Bildhauers in dem Vergleiche mit der das Vorbild getreuer wiedergebenden Zeichnung gefunden haben. Das ihm Eigene ist die sinnige und poetische Auffassung, die er ein Dichter für die Todte empfunden und die er in der feinen Mischung von Wehmuth und Glück, von Lebenshoffnung und Todeswirklichkeit auf die Züge des Mädchens übertragen. Louis Gonse hat es ausgesprochen - sie ist eine Schwester der Gioconda! So verschieden der Ausdruck und der Charakter in beiden, die subjective Stimmung, aus der heraus sie Leben erbielten, ist eine verwandte und Verrocchios Werkstatt war es, welche dieselbe zeitigte. Was an Raphael erinnerte und Springer veranlasste, das Werk in seiner Biographie desselben zu 
besprechen, waren die Formen, deren Eigenthümlichkeit, wie wir gesehen, auf eine andere, nämlich antike Quelle zurückzuführen ist -der Ausdruck kam rou einer Raphael fremden Richtung, derjenigen, die mit Lionardos Namen gekennzeichnet wird. Und dieses war es, was Alexander Dumas Sohn, in dessen Arbeitszimmer eine getreue Copie des Kopfes Platz gefunden, bestimmte, in einem ron Gonse theilweise publicirten Briefe einem Freund zu schreiben: „moi, je la crois de Léonard, mais mon opinion ue fait rien à l'affaire". Lionardos Autorschaft behaupten ist freilich zu gewagt, aber dem Kreise, aus dem er herrorgegangen. steht die Büste nahe.

Und gerade von Verrocechio erzählt uns Vasari ${ }^{1}$ ), dass er einer der ersten war, der Gypsabgüsse ron Leichen nabm, die Kunst des Modellirens in Wachs selbst betrieb und den durch sie später am berühmtesten gewordenen Meister Orsino Benitendi in dieselbe einweihte. Wenn Louis Gonse auf diese Angaben bin den Liller Kopf dem letzteren zuweist, so kann das allerdings, so lange uns keine sonstigen Werke desselben bekaunt sind, nur Hypothese bleiben. Es scheint mir daher gerathener, da sichere Beweisgründe fehlen, bei dem allgemeineren Resultat zu verharren und ohne neue Künstleruamen in Vorschlag zu bringen, die Beziehung zu Verrocchio und seiner Schule wiederholt za betonen. Ob der Bildhauer zugleich der Verfertiger der Zeichnung gewesen, muss in gleicher Weise eine offene Frage bleiben. Für Raphael spricht eben nichts, als der ihm in gewisser Art verwandte Charakter des Kopfes und dieser kann als getreu wiedergegebenes Vorbild ni.ht massgebend sein.

Im Uebrigen aber finden sich $z u$ wenige ausschlaggebende Anzeichen, als dass man ausschliesslich auf technische Eigenthümlichkeiten Rücksicht nehmend einen bestimmten Meister nennen könnte. Wer immer aber die Künstler gewesen sein mögen - und es gelingt dereinst vielleicht doch noch sie festzustellen - es ist ihnen geglückt, den räthselhaft bezaubernden Eindruck, unter dem sie selbst gestanden, in späteren Zeiten Nachgeborenen mitzutheilen und ist es für uns auch nicht mehr "Julia, die Tochter Cicero's", so doch ein bestrickendes Traumbild, in dem uns Alterthum und Renaissance Eines zu werden scheineu, ein unlösliches und vollendetes Ganze, wie es die Menschen geträumt zur Zeit, als sie zum Capitol strömten, die wiedererstandene Römerin zu begrüssen!

1) Ausgabe Milanesi T. HI, ১. :, i: ff. 